\title{
GRANULOMATOSIS WITH POLYANGIITIS MIMETIZING RHEUMATOID VASCULITIS
}

Rita Marina Soares de Castro Duarte (Hospital Universitário da Universidade Federal do Vale do São Francisco, Petrolina, PE, Brasil), David Lopes Lima Cavalcanti Coelho (Hospital Universitário da Universidade Federal do Vale do São Francisco, Petrolina, PE, Brasil), Caroline Diniz Bezerra (Hospital Universitário da Universidade Federal do Vale do São Francisco, Petrolina, PE, Brasil), Alexsandro Paulo Costa Galdino Junior (Hospital Universitário da Universidade Federal do Vale do São Francisco, Petrolina, PE, Brasil), Leonardo Fernandes e Santana (Hospital Universitário da Universidade Federal do Vale do São Francisco, Petrolina, PE, Brasil), Mateus de Sousa Rodrigues (Hospital Universitário da Universidade Federal do Vale do São Francisco, Petrolina, PE, Brasil), Luana Barros Caxias de Souza (Hospital Universitário da Universidade Federal do Vale do São Francisco, Petrolina, PE, Brasil), Bianca Alencar Dias Almeida (Hospital Universitário da Universidade Federal do Vale do São Francisco, Petrolina, PE, Brasil)

\section{BACKGROUND}

Granulomatosis with polyangiitis (GPA) is a pauci-immune necrotizing granulomatous vasculitis, which affects predominantly small vessels, mainly from the respiratory tract and kidneys. It has a broad spectrum of clinical manifestations that mimics other diseases, such as: Pyoderma gangrenosum, lymphoma, tuberculosis, sarcoidosis, subacute bacterial endocarditis and systemic lupus erythematosus. This case report aims to highlight the differential diagnosis of GPA with rheumatoid vasculitis.

\section{CASE REPORT}

MMSM, female, 53 years old. Presents with a history of rheumatoid arthritis 3 years ago, hypothyroidism, recurrent rhinosinusitis, and reduced hearing acuity bilaterally. Referred to the rheumatology outpatient clinic for the control of rheumatoid arthritis. In chronic use of leflunomide and prednisone. The patient had begun treatment with tuberculosis scheme (rifampicin, isoniazid, ethambutol, pyrazinamide) 4 months ago in another service. During consultation, she reported dyspnea on mild efforts, hemoptysis, and paresthesia in both soles. Physical examination noticed ulcerated lesions with hyperemic borders, on both lateral malleolus, nodules with ulcerated center on elbows and splinter hemorrhages. Normal pulmonary and cardiac auscultation. Hands without synovitis or deformities. The patient had viral serologies negative (HIV, hepatitis B and C), normal urine summary and proteinuria, positive rheumatoid factor at low titles and negative anti-CCP. Chest tomography showing nodular lesions in both lungs, some of these with cavitation. Research of Acid-Alcohol-Resistant Bacilli (AARB) was negative in bronchoalveolar lavage. Patient was hospitalized for diagnostic investigation. The laboratory tests evidenced leukocytosis with neutrophilia, normal vitamin B12, negative blood cultures, negative anti-CCP, negative FR, negative p-ANCA, positive c-ANCA 1:80, urine summary with mild hematuria (10 red cells per field). Radiography of the hands showed no erosion. Transesophageal echocardiogram showed no vegetations. Nasoscopy showed bilaterally ulcerated lesion with nasal septum crusts. Patient met EMA (European Medicines Agency) criteria for GPA, which suggest the diagnosis in the absence of biopsy. Remission induction therapy with methylprednisolone and cyclophosphamide pulses was initiated, in addition to oral analgesia and trimethoprim- sulfamethoxazole. It progressed with clinical improvement and healing of skin lesions. Currently, the outpatient remission induction scheme is maintained.

\section{CONCLUSION}

The clinical presentation of GPA, in this case, makes a differential diagnosis with rheumatoid vasculitis. Upper airway involvement, reduced hearing acuity, and cavitary lung lesions, without regression to the established treatment, suggest the diagnosis of GPA. Differently, a rheumatoid vasculitis occurs in patients 
with long-standing seropositive rheumatoid arthritis. Histologically, GPA lesions affect small vessels with necrotic granulomas, while rheumatoid vasculitis courses with leukocytoclastic vasculitis. 